\title{
High Carbohydrate Diet Is Associated with Severe Clinical Indicators, but Not with Nutrition Knowledge Score in Patients with Multiple Myeloma
}

\author{
Ema Borsi ${ }^{1}$, Costela Lacrimioara Serban ${ }^{2, *} \mathbb{0}$, Cristina Potre ${ }^{1,3}$, Ovidiu Potre ${ }^{1,3}$, Salomeia Putnoky ${ }^{4, *}$, \\ Miruna Samfireag ${ }^{3,5}$, Raluca Tudor ${ }^{6}\left(\mathbb{D}\right.$, Ioana Ionita ${ }^{1,3}\left(\mathbb{D}\right.$ and Hortensia Ionita ${ }^{1,3}$
}

1 Discipline of Hematology, Department of Internal Medicine, Victor Babes University of Medicine and Pharmacy, 300041 Timisoara, Timis County, Romania; borsi.ema@umft.ro (E.B.); potre.cristina@umft.ro (C.P.); potre.ovidiu@umft.ro (O.P.); ionita.ioana@umft.ro (I.I.); ionitahortensia@gmail.com (H.I.)

2 Department of Functional Science, Victor Babes University of Medicine and Pharmacy, 300041 Timisoara, Timis County, Romania

3 Hematology Clinic, Timisoara's Emergency City Hospital, 300723 Timisoara, Timis County, Romania; samfireag.miruna@umft.ro

4 Department of Microbiology, Victor Babes University of Medicine and Pharmacy, 300041 Timisoara, Timis County, Romania; putnoky.salomeia@umft.ro

5 Department of Internal Medicine, Discipline of Clinical Practical Skills, Victor Babes University of Medicine and Pharmacy, 300041 Timisoara, Timis County, Romania

Citation: Borsi, E.; Serban, C.L.;

Potre, C.; Potre, O.; Putnoky, S.;

Samfireag, M.; Tudor, R.; Ionita, I.;

Ionita, H. High Carbohydrate Diet Is Associated with Severe Clinical

Indicators, but Not with Nutrition

Knowledge Score in Patients with

Multiple Myeloma. Int. J. Environ.

Res. Public Health 2021, 18, 5444.

https://doi.org/10.3390/

ijerph18105444

\section{Academic Editors: Krystyna}

Gutkowska, Dominika Głąsska and Dominika Guzek

Received: 18 April 2021

Accepted: 17 May 2021

Published: 19 May 2021

Publisher's Note: MDPI stays neutral with regard to jurisdictional claims in published maps and institutional affiliations.

6 Department of Neurology, Victor Babes University of Medicine and Pharmacy, 300041 Timisoara, Timis County, Romania; tudor.raluca@umft.ro

* Correspondence: costela.serban@umft.ro (C.L.S.); putnoky.salomeia@umft.ro (S.P.); Tel.: +40-770-198-363 (C.L.S.)

\begin{abstract}
Although the survival rate of patients diagnosed with multiple myeloma has doubled over the last few decades, due to the introduction of new therapeutic lines and improvement of care, other potential contributors to the therapeutic response/relapse of disease, such as nutrient intake, along with nutrition knowledge, have not been assessed during the course of the disease. The purpose of this research was to assess nutrition knowledge and diet quality in a group of patients with a diagnosis of multiple myeloma. Anthropometric, clinical and biological assessments and skeletal survey evaluations, along with the assessment of nutritional intake and general nutrition knowledge, were performed on 61 patients with a current diagnosis of multiple myeloma. A low carbohydrate diet score was computed, classified in tertiles, and used as a factor in the analysis. Patients in tertiles indicative of high carbohydrate or low carbohydrate intake showed significant alteration of clinical parameters, such as hemoglobin, uric acid, albumin, total proteins, beta-2 microglobulin, percentage of plasmacytes in the bone marrow and D-dimers, compared to patients in the medium carbohydrate intake tertile. Nutrition knowledge was not associated with clinical indicators of disease status, nor with patterns of nutrient intake. Better knowledge of food types and nutritional value of foods, along with personalized nutritional advice, could encourage patients with MM to make healthier decisions that might extend survival.
\end{abstract}

Keywords: multiple myeloma; nutrition knowledge; nutrients; diet; clinical outcome

\section{Introduction}

In 2020, the global age-standardized incidence rates for multiple myeloma (MM) were 2.2/100,000 for males and 1.5/100,000 for females, with an age-standardized mortality rate of 1.1/100,000 [1]. Recognized and relatively constant risk factors are older age, male gender and African ethnicity [2]. Due to new therapies and constant improvements in care, survival has more than doubled over recent decades. Recently, Usmani et al. [3] recognized that in newly diagnosed transplant eligible $\mathrm{MM}$ patients, age ( $<65$ years), non-IgA isotype, normal albumin levels, low beta-2 microglobulin $\leq 3.5 \mathrm{mg} / \mathrm{dl}$, serum 
creatinine levels $<2 \mathrm{mg} / \mathrm{dL}$, hemoglobin levels $\geq 10 \mathrm{~g} / \mathrm{dL}$ and platelet count $\geq 150 \mathrm{k} / \mu \mathrm{L}$ had a positive effect on the 10-year survival, but elevated serum LDH levels and any cytogenetic abnormalities did not negatively predict 10 -year survival. Still, the survival of patients with MM is impaired, with only $10-15 \%$ reaching the lifespan of the general population [3].

Recently, the relationship between diet and the risk of $\mathrm{MM}$, as well as the relationship between diet and prognosis of patients with $\mathrm{MM}$, has been tackled in several publications; however, most of the relationships that were studied pertained to pre-diagnosis diet. Protective factors for MM in adulthood included increased intake of fruits (over three servings per week) [4], increased intake of fish [5] and current alcohol intake in both genders compared to non-drinkers [6]. Risk factors for MM included the intake of more than one serving of artificially sweetened drinks [7]. Lee et al. [8] determined that an unhealthy overall diet pre-diagnosis is associated with poor survival rates in patients with MM.

Although the relationship between nutrition knowledge, food choices and food intake is complex, little is known about the level of nutrition knowledge in patients diagnosed with MM and the quality of their diets after diagnosis. Our aim was to assess the nutritional knowledge and quality of diets in a cohort of patients with $\mathrm{MM}$, and prepare an intervention study to increase general and specific nutrition knowledge, including nutrition counseling.

\section{Materials and Methods}

\subsection{Participants and Samples}

From a cohort of 76 patients followed up in the Hematology Department of the Municipal Emergency Hospital, Timisoara, Romania, 61 participants were included in this research (Figure 1). Patients were recruited between September 2020 and January 2021, during the second wave of the COVID-19 pandemic. This research was designed as a crosssectional investigation pilot study. Nevertheless, the sample size was sufficient, taking into account a minimum sample of 59 participants, calculated by Viechtbauer et al. [9] for pilot studies.

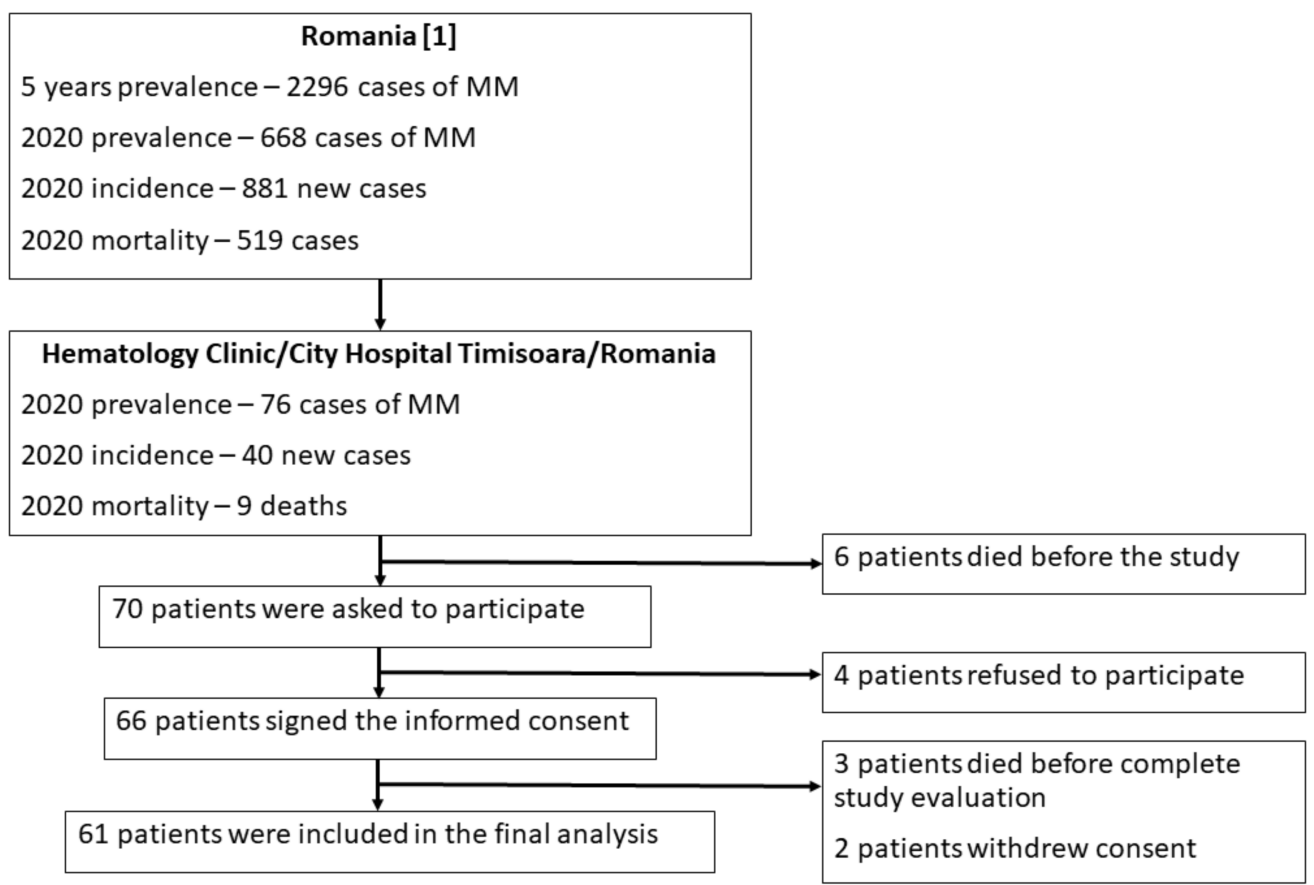

Figure 1. Study flow chart. 
Diagnosis, staging and therapy were established using ESMO clinical practice guidelines [10]. Data were collected during follow-up visits, and included anthropometric, hematological, biochemical and immunological parameters, radiological data, whole body MRI and type of and response to treatment, as well as survival duration in months (from the time of diagnosis up to the end of the study). For this pilot study, the following inclusion criteria were used: age $>18$ years, diagnosis of MM in clinical stage 1-3 and a follow-up of at least 6 months, according to the protocol. For the purposes of analysis, the exclusion criteria were set as follows: allogeneic SCT, monoclonal gammopathy of undetermined significance and drug-naïve patients. The exclusion criteria also took into consideration the inability to provide informed consent, and the inability to provide accurate anamnestic data.

Patients were included only after signing the informed consent. The entire study was conducted according to the principles stated in the Declaration of Helsinki, and was approved by the Ethics Committee of the "Victor Babes" University of Medicine and Pharmacy, Timisoara, Romania, with no. 6/2019.

\subsection{Clinical and Biological Evaluation}

Height and weight were measured as per the international guidelines, and were used for the calculation of body mass index $\left(\mathrm{BMI} ; \mathrm{BMI}=\right.$ weight $(\mathrm{kg}) /$ height $\left.(\mathrm{m})^{2}\right)$. The nutritional status of the participants was determined using the following BMI thresholds: underweight (BMI below $\left.18.5 \mathrm{~kg} / \mathrm{m}^{2}\right)$, normal weight $\left(18.5-24.9 \mathrm{~kg} / \mathrm{m}^{2}\right)$, overweight $\left(25-29.9 \mathrm{~kg} / \mathrm{m}^{2}\right.$ ) and obese (over $30 \mathrm{~kg} / \mathrm{m}^{2}$ ). All patients underwent whole-body low-dose computed tomography. All blood samples were collected in the morning. Complete blood count implied the collection of venous blood in a sterile EDTA vacutainer, using fluorescence flow cytometry. Coagulation tests consisted of the following: dosing of fibrinogen through a coagulometric method, and performing quantitative tests to dose D-dimer; analysis of venous blood collected in a vacutainer with sodium citrate, with plasma separation by centrifugation, and processing using a latex method through automatic agglutination with photometric detection. The samples for serum blood urea nitrogen, creatinine, calcium and LDH testing were collected in a sterile vacutainer without anticoagulant, with/without gel separator, by a spectrophotometric technique. Beta- 2 microglobulin was assessed using the nephelometric method. The samples underwent analysis via serum protein electrophoresis with immunofixation, serum-free light chain quantification, heavy/light chain quantification, immunoglobulin and total proteins. All patients underwent bone marrow aspiration.

The diagnosis of chronic kidney disease was established by measuring serum creatinine levels, in order to calculate the glomerular filtration rate, and by measuring the urinary albumin/creatinine ratio, in order to detect proteinuria [11]. Osteoporosis was diagnosed based on bone mineral density determination obtained from dual energy $\mathrm{X}$-ray absorptiometry assessment [12]. Major depression was diagnosed by a psychiatrist, using the DSM-5 criteria [13], and clinical assessments were conducted in order to diagnose any potential peripheral neuropathy. Information about diarrhea/constipation was obtained during the anamnestic process, and information about any infections was extracted from clinical records.

\subsection{Nutritional Assessment}

Dietary intake assessment was performed using a validated FFQ, consisting of 53 food items; it investigated food intake during the last 30 days [14,15]. For each item, the frequency and the usual amount of consumed food items were investigated. To give an estimate of the quantity of fat or added sugar, additional questions were asked in relation to some of the items. The intakes were converted to grams, in accordance with the guidelines of household scales [16]. Using a computer program specially created for this purpose, the energy and macronutrient intakes were calculated for each individual. Macronutrients were transformed into a percentage of contribution to total energy, and then the adequacy of intake was calculated as per the European Food and Safety Authority 
(EFSA) recommendations [17]. "Inadequate intake" had different meanings for different macronutrients: for proteins, "inadequate" meant lower than the recommended values; for fat, "inadequate" represented only the values above the upper recommended threshold; as for carbohydrates, the values were both lower and upper thresholds. The energy-adjusted values of intake were also computed for saturated fat, fibers and alcohol.

A low carbohydrate diet (LCD) score was computed using deciles of the percentage of macronutrients. For carbohydrates, the lowest decile received a score of 10, and the highest decile received a score of one. For fat and protein, the lowest decile received a score of one, and the highest received a score of 10. All individual macronutrient scores were added to obtain the LCD score, which ranged from a possible lowest score of 3 to a highest score of 30, with higher scores indicating higher adherence to a low carbohydrate diet $[18,19]$. The LCD score was transformed into tertiles and further used as a three-layer factor in statistical analysis.

\subsection{Nutrition Knowledge Assessment}

A previously validated questionnaire [20] was used to assess nutrition knowledge. The nutritional knowledge assessment included 88 items. Each item allowed for only one answer, and the obtained points were added for each correct answer provided by the responders, in order to determine the sub-score per section and the total knowledge score.

\subsection{Statistical Analysis}

Statistical analysis was performed using the Statistical Package for Social Sciences (SPSS Corp, version 18, Chicago, IL, USA). Frequency and percentage were used for the description of categorical data, and mean and standard deviation were used for continuous data. The normality of data was evaluated by the Shapiro-Wilk test. All continuous variables were normally distributed, therefore parametric tests ( $t$-test and ANOVA) were employed for the comparisons of means between the categories. Chi-square or MannWhitney or Kruskal-Wallis tests were used to compare the characteristics of participants for categorical data.

\section{Results}

Within the group, $13.1 \%$ (8) of participants were in disease stage 1, 21.3\% (13) were in disease stage 2 and $65.6 \%$ (40) were in disease stage 3 . Females represented $57.4 \%$. Mean age was $65.2+/-9.5$ years, within the age range of $41-84$ years. The mean BMI was $26.2+/-4.2 \mathrm{~kg} / \mathrm{m}^{2}$, with $57.4 \%$ (35) being overweight and obese, and the remaining $42.6 \%$ (26) having a weight within normal limits. In Table 1, the clinical features of the cohort of patients diagnosed with MM are presented using disease stage as a factor. The proportions of genders, kappa or lambda light chains in blood and urine, the presence of infections, increased viscosity, depression and therapeutic response did not statistically differ between stages 1 and 2 versus stage 3 . Additionally, mean age, BMI and number of therapeutic lines were not statistically different between the two categories. All the rest of the clinical variables showed statistical differences between stages 1 and 2 versus stage 3 .

Unadjusted energy ( $\mathrm{kcal})$, macronutrient intake $(\mathrm{g})$ and total sugar $(\mathrm{g})$, fiber $(\mathrm{g})$ and alcohol (g) by tertiles of energy are presented in Table 2. Table 2 also contains the energyadjusted macronutrients (as a percentage to energy contribution), and adjusted sugar, fiber and alcohol per $1000 \mathrm{kcal}$ of intake. While unadjusted intakes are statistically different, the adjusted intakes are not statistically different between tertiles of energy. A lower carbohydrate diet score is observed in the first tertile of energy, as compared to other tertiles. A linear trend of the adequacy of intake was observed for proteins, but not for fat or carbohydrates. 
Table 1. Clinical features of the cohort of patients with a diagnosis of $\mathrm{MM}$ by disease staging $(\mathrm{N}=61)$.

\begin{tabular}{|c|c|c|c|c|c|}
\hline Clinical Features & Measure & $\begin{array}{c}\text { Disease Stages } 1 \\
\text { and } 2(n 1=21)\end{array}$ & $\begin{array}{l}\text { Disease Stage } \\
3(\mathrm{n} 2=40)\end{array}$ & $p$-Value & Total \\
\hline Sex F & $\mathrm{n}(\%) *$ & $12(57.1 \%)$ & $23(57.5 \%)$ & 0.979 & $35(57.4 \%)$ \\
\hline Age (years) & Mean +/-SD $* *$ & $65.1+/-11.7$ & $65.2+/-8.2$ & 0.964 & $65.2+/-9.5$ \\
\hline $\begin{array}{l}\text { Body mass index } \\
\qquad\left(\mathrm{kg} / \mathrm{m}^{2}\right)\end{array}$ & Mean +/ - SD ** & $26.3+/-5.1$ & $26.2+/-3.7$ & 0.917 & $26.3+/-4.2$ \\
\hline $\begin{array}{l}\text { Follow-up since } \\
\text { diagnosis (months) }\end{array}$ & Mean +/ - SD ** & $32.9+/-19.7$ & $22.1+/-14.3$ & 0.018 & $25.8+/-17.0$ \\
\hline $\begin{array}{l}\text { Blood smear } \\
\text { alterations }\end{array}$ & $\mathrm{n}(\%) *$ & $7(33.3 \%)$ & $34(85.0 \%)$ & $<0.001$ & $41(67.2 \%)$ \\
\hline Anemia & $\mathrm{n}(\%) *$ & $15(71.4 \%)$ & $40(100.0 \%)$ & 0.001 & $55(90.2 \%)$ \\
\hline Hemoglobin (g/dl) & Mean +/ - SD ** & $10.9+/-1.7$ & $7.3+/-0.9$ & $<0.001$ & $8.6+/-2.1$ \\
\hline Hypercalcemia & $\mathrm{n}(\%)^{*}$ & $6(28.6 \%)$ & $26(65.0 \%)$ & 0.007 & $32(52.5 \%)$ \\
\hline $\begin{array}{l}\text { Serum calcium } \\
(\mathrm{mg} / \mathrm{dL})\end{array}$ & Mean +/ - SD ** & $10.6+/-2.3$ & $12.8+/-3.1$ & 0.004 & $12.0+/-3.0$ \\
\hline $\begin{array}{c}\text { Alkaline Phosphatase } \\
\text { (IU/L) }\end{array}$ & Mean +/-SD ** & $71.9+/-18.5$ & $116.0+/-36.9$ & $<0.001$ & $100.8+/-38.0$ \\
\hline $\begin{array}{c}\text { Beta-2 microglobulin } \\
\geq 3.5\end{array}$ & $\mathrm{n}(\%) *$ & $13(61.9 \%)$ & $40(100.0 \%)$ & $<0.001$ & $53(86.9 \%)$ \\
\hline $\begin{array}{l}\text { Beta-2 microglobulin } \\
(\mathrm{mg} / \mathrm{dl})\end{array}$ & Mean +/-SD ** & $3.7+/-0.9$ & $11.2+/-3.1$ & $<0.001$ & $8.6+/-4.4$ \\
\hline $\begin{array}{c}\text { Percentage of } \\
\text { plasmacytes }>60 \%\end{array}$ & $\mathrm{n}(\%) *$ & $3(14.3 \%)$ & $28(70.0 \%)$ & $<0.001$ & $31(50.8 \%)$ \\
\hline $\begin{array}{c}\text { Plasmacytes in bone } \\
\text { marrow }(\%)\end{array}$ & Mean +/-SD ** & $36.8+/-20.6$ & $68.1+/-16.2$ & $<0.001$ & $57.3+/-23.2$ \\
\hline Uric acid (mg/dL) & Mean +/-SD ** & $5.1+/-1.4$ & $7.5+/-1.8$ & $<0.001$ & $6.7+/-2.0$ \\
\hline Creatinine (mg/dL) & Mean +/ $-\mathrm{SD}^{* *}$ & $1.3+/-0.9$ & $2.5+/-1.3$ & $<0.001$ & $2.1+/-1.3$ \\
\hline $\begin{array}{l}\text { Blood urea nitrogen } \\
(\mathrm{mg} / \mathrm{dL})\end{array}$ & Mean +/ - SD ** & $42.4+/-17.3$ & $67.3+/-32.3$ & $<0.001$ & $58.7+/-30.4$ \\
\hline Albumin (g/L) & Mean +/-SD ${ }^{* *}$ & $59.7+/-5.7$ & $51.1+/-3.9$ & $<0.001$ & $54.0+/-6.1$ \\
\hline Total protein $(\mathrm{g} / \mathrm{dL})$ & Mean +/-SD $* *$ & $6.6+/-0.9$ & $9.2+/-3.8$ & $<0.001$ & $8.3+/-3.3$ \\
\hline $\begin{array}{c}\text { Erythrocyte } \\
\text { sedimentation rate } \\
(\mathrm{mm} / \mathrm{hr})\end{array}$ & Mean +/-SD ** & $87.8+/-40.9$ & $114.6+/-38.5$ & 0.014 & $105.4+/-41.1$ \\
\hline $\begin{array}{c}\text { C-reactive protein } \\
(\mathrm{mg} / \mathrm{L})\end{array}$ & Mean +/-SD ** & $8.5+/-9.8$ & $21.7+/-14.4$ & $<0.001$ & $17.2+/-14.4$ \\
\hline $\mathrm{LDH}(\mathrm{U} / \mathrm{L})$ & Mean +/ $-\mathrm{SD}^{* *}$ & $226.0+/-63.0$ & $359.5+/-121.8$ & $<0.001$ & $313.6+/-122.7$ \\
\hline Fibrinogen (mg/dL) & Mean +/-SD $* *$ & $421.4+/-123.6$ & $507.5+/-152.6$ & 0.030 & $477.8+/-148.1$ \\
\hline D-dimers (ng/mL) & Mean $+/-\mathrm{SD}^{* *}$ & $220.7+/-59.7$ & $340.4+/-118.4$ & $<0.001$ & $299.1+/-116.5$ \\
\hline \multirow{2}{*}{$\begin{array}{l}\text { Serum-free light } \\
\text { chain }\end{array}$} & Kappa $\mathrm{n}$ & $16(76.2 \%)$ & $26(65.0 \%)$ & \multirow{2}{*}{0.370} & $42(68.9 \%)$ \\
\hline & Lambda & $5(23.8 \%)$ & $14(35.0 \%)$ & & $19(31.1 \%)$ \\
\hline \multirow{3}{*}{$\begin{array}{l}\text { Immunoglobulin } \\
\text { type }\end{array}$} & $\operatorname{IgG}$ & $15(71.4 \%)$ & $23(57.5 \%)$ & \multirow{3}{*}{0.429} & $38(62.3 \%)$ \\
\hline & $\mathrm{n}(\%)^{*}$ & $5(23.8 \%)$ & $16(40.0 \%)$ & & $21(34.4 \%)$ \\
\hline & $\operatorname{IgM}$ & $1(4.8 \%)$ & $1(2.5 \%)$ & & $2(3.3 \%)$ \\
\hline
\end{tabular}


Table 1. Cont.

\begin{tabular}{|c|c|c|c|c|c|}
\hline Clinical Features & Measure & $\begin{array}{c}\text { Disease Stages } 1 \\
\text { and } 2(\mathrm{n} 1=21)\end{array}$ & $\begin{array}{c}\text { Disease Stage } \\
3(\mathrm{n} 2=40)\end{array}$ & $p$-Value & Total \\
\hline Infections & $\mathrm{n}(\%) *$ & $2(9.5 \%)$ & $7(17.5 \%)$ & 0.479 & $9(14.8 \%)$ \\
\hline Myelosuppression & $\mathrm{n}(\%) *$ & $0(0.0 \%)$ & $10(25.0 \%)$ & 0.011 & $10(16.4 \%)$ \\
\hline $\begin{array}{c}\text { Chronic kidney } \\
\text { disease }\end{array}$ & $\mathrm{n}(\%) *$ & $3(14.3 \%)$ & $21(52.5 \%)$ & 0.004 & $24(39.3 \%)$ \\
\hline $\begin{array}{l}\text { Peripheral } \\
\text { neuropathy }\end{array}$ & $\mathrm{n}(\%) *$ & $0(0.0 \%)$ & $31(77.5 \%)$ & $<0.001$ & $31(50.8 \%)$ \\
\hline Osteoporosis & $\mathrm{n}(\%) *$ & $1(4.8 \%)$ & $17(42.5 \%)$ & 0.002 & $18(29.5 \%)$ \\
\hline Depression & $\mathrm{n}(\%) *$ & $14(66.7 \%)$ & $19(47.5 \%)$ & 0.153 & $33(54.1 \%)$ \\
\hline Constipation/diarrhea & $\mathrm{n}(\%) *$ & $0(0.0 \%)$ & $16(40.0 \%)$ & 0.001 & $16(26.2 \%)$ \\
\hline $\begin{array}{l}\text { Autologous stem cell } \\
\text { transplantation }\end{array}$ & $\mathrm{n}(\%) *$ & $0(0.0 \%)$ & $9(22.5 \%)$ & 0.021 & $9(14.8 \%)$ \\
\hline \multirow{4}{*}{ Response } & Partial remission & $8(38.1 \%)$ & $15(37.5 \%)$ & \multirow{4}{*}{0.370} & $23(37.7 \%)$ \\
\hline & Total remission & $9(42.9 \%)$ & $10(25.0 \%)$ & & $19(31.1 \%)$ \\
\hline & Stable disease & $3(14.3 \%)$ & $9(22.5 \%)$ & & $12(19.7 \%)$ \\
\hline & Progressive disease & $1(4.8 \%)$ & $6(15.0 \%)$ & & $7(11.5 \%)$ \\
\hline $\begin{array}{l}\text { Therapeutic lines } \\
\text { administered }\end{array}$ & Mean $+/-\mathrm{SD}^{* * *}$ & $3.2+/-1.4$ & $2.8+/-1.6$ & 0.247 & $2.9+/-1.5$ \\
\hline
\end{tabular}

Notes: Data are presented as mean $+/-\mathrm{SD}$, or as $\mathrm{n}(\%)$, as appropriate. ${ }^{*}$ Chi-square test, ${ }^{* *}$ independent samples $t$-test, ${ }^{* * *}$ Mann-Whitey test. $p$-values in bold are statistically significant. Abbreviations: $\mathrm{SD}=$ standard deviation, $\mathrm{n}=$ number of participants.

Table 2. Nutrient intake per tertile of energy intake $(\mathrm{N}=61)$.

\begin{tabular}{|c|c|c|c|c|c|}
\hline \multirow{2}{*}{ Nutrient Intake } & \multirow{2}{*}{ Measure } & \multicolumn{3}{|c|}{ Tertiles of Energy (kcal) Intake } & \multirow[t]{2}{*}{$\begin{array}{l}p \text {-Value for } \\
\text { Trend }\end{array}$} \\
\hline & & First $(n 1=20)$ & Second $(\mathrm{n} 2=21)$ & Third $(\mathrm{n} 3=20)$ & \\
\hline Energy (kcal) & Mean +/-SD * & $797.6+/-139.2$ & $1259.1+/-164.0$ & $\begin{array}{c}2296.0+/- \\
1030.9\end{array}$ & $<0.001$ \\
\hline Protein $(\mathrm{g})$ & Mean +/-SD* & $39.7+/-9.1$ & $54.8+/-11.8$ & $92.9+/-34.9$ & $<0.001$ \\
\hline Fat total (g) & Mean +/-SD* & $31.1+/-6.9$ & $44.8+/-11.5$ & $80.9+/-34.3$ & $<0.001$ \\
\hline Carbohydrate (g) & Mean $+/-S^{*}$ & $89.4+/-20.2$ & $161.6+/-36.8$ & $301.5+/-188.4$ & $<0.001$ \\
\hline Saturated fat (g) & Mean $+/-S^{*}$ & $10.1+/-2.2$ & $15.7+/-4.8$ & $27.0+/-9.9$ & $<0.001$ \\
\hline Fiber total dietary $(\mathrm{g})$ & Mean +/-SD * & $6.9+/-2.2$ & $13.8+/-6.5$ & $25.0+/-25.7$ & 0.002 \\
\hline Alcohol (g) & Mean $+/-\mathrm{SD}^{*}$ & $1.2+/-2.0$ & $1.2+/-1.8$ & $3.1+/-3.6$ & 0.036 \\
\hline Percentage of energy from fat & Mean $+/-\mathrm{SD}^{*}$ & $35.0+/-4.7$ & $31.9+/-6.3$ & $32.7+/-9.3$ & 0.361 \\
\hline $\begin{array}{l}\text { Percentage of energy from } \\
\text { carbohydrates }\end{array}$ & Mean +/-SD* & $44.8+/-6.6$ & $51.3+/-9.3$ & $50.8+/-12.3$ & 0.057 \\
\hline Saturated fat (g)/1000 kcal & Mean $+/-\mathrm{SD}^{*}$ & $1.3+/-0.1$ & $1.2+/-0.3$ & $1.2+/-0.4$ & 0.946 \\
\hline Total fiber $(\mathrm{g}) / 1000 \mathrm{kcal}$ & Mean $+/-\mathrm{SD}^{*}$ & $8.6+/-2.5$ & $10.9+/-4.7$ & $9.9+/-5.3$ & 0.237 \\
\hline Alcohol (g)/1000 kcal & Mean +/-SD * & $1.5+/-2.7$ & $1.0+/-1.4$ & $1.4+/-1.8$ & 0.648 \\
\hline LCD score & $\underset{* *}{\operatorname{Mean}+/-\mathrm{SD}}$ & $19.9+/-5.8$ & $14.7+/-7.4$ & $15.0+/-8.3$ & 0.045 \\
\hline Protein adequate intake $\mathrm{n}(\%)$ & $\mathrm{n}(\%)^{* *}$ & $7(35.0 \%)$ & $14(66.7 \%)$ & $20(100.0 \%)$ & $<0.001$ \\
\hline Fat adequate intake $\mathrm{n}(\%)$ & $\mathrm{n}(\%) * *$ & $10(50.0 \%)$ & $16(76.2 \%)$ & $11(55.0 \%)$ & 0.193 \\
\hline
\end{tabular}


Table 2. Cont.

\begin{tabular}{cccccc}
\hline \multirow{2}{*}{ Nutrient Intake } & Measure & \multicolumn{2}{c}{ Tertiles of Energy (kcal) Intake } & $\begin{array}{c}p \text {-Value for } \\
\text { Trend }\end{array}$ \\
\cline { 4 - 6 } & & First (n1 = 20) & Second (n2 = 21) & Third (n3 = 20) \\
\hline \multirow{2}{*}{$\begin{array}{c}\text { Carbohydrate } \\
\text { intake }\end{array}$} & $\begin{array}{c}\text { Below adequate } \\
\mathrm{n}(\%)\end{array}$ & $\mathrm{n}(\%)^{* *}$ & $9(45.0 \%)$ & $6(28.6 \%)$ & $7(35.0 \%)$ \\
\cline { 2 - 5 } & Adequate $\mathrm{n}(\%)$ & $\mathrm{n}(\%)^{* *}$ & $11(55.0 \%)$ & $11(52.4 \%)$ & $7(35.0 \%)$ \\
\cline { 2 - 5 } & $\begin{array}{c}\text { Over adequate } \\
\mathrm{n}(\%)\end{array}$ & $\mathrm{n}(\%)^{* *}$ & $0(0.0 \%)$ & $4(19.0 \%)$ & $6(30.0 \%)$ \\
\hline
\end{tabular}

Notes: Data are presented as mean $+/-\mathrm{SD}$, or as $\mathrm{n}(\%)$, as appropriate. ${ }^{*} \mathrm{ANOVA},{ }^{* *}$ Kruskall-Wallis test; $p$-values in bold are statistically significant. Abbreviations: $\mathrm{SD}=$ standard deviation, $\mathrm{n}=$ number of participants, $\mathrm{LCD}=$ low carbohydrate diet.

In Table 3, the demographic and clinical features are presented by tertiles of low carbohydrate diet scores, with the first tertile indicating higher carbohydrate intake, the second tertile indicating medium carbohydrate intake and the third tertile indicating low carbohydrate intake. Females are more likely than men to report a high carbohydrate diet. The low carbohydrate tertile has significantly higher proportions of higher than adequate fat intake, as compared to medium carbohydrate tertile. Energy-adjusted saturated fat, total fiber and alcohol show significant differences between tertiles of the LCD score. Other clinical characteristics that show differences between tertiles of the LCD score include hemoglobin, uric acid, albumin, total proteins, beta- 2 microglobulin, percentage of plasmacytes in the bone marrow and D-dimers. Clinical indicators, namely types of response to therapy and the number of therapeutic lines, are not associated with tertiles of the LCD score.

Table 3. Clinical and intake variables per tertile of LCD score.

\begin{tabular}{|c|c|c|c|c|c|c|}
\hline \multirow{2}{*}{\multicolumn{2}{|c|}{ Clinical and Intake Variables }} & \multirow[b]{2}{*}{ Measure } & \multicolumn{3}{|c|}{ Tertiles Of LCD Score } & \multirow[t]{2}{*}{$p$-Values } \\
\hline & & & $\begin{array}{l}\text { First (High Carb } \\
\text { Diet) } n 1=21\end{array}$ & $\begin{array}{c}\text { Second } \\
\text { (Medium Carb } \\
\text { Diet) } n 2=18\end{array}$ & $\begin{array}{l}\text { Third (Low Carb } \\
\text { Diet) n3 = } 22\end{array}$ & \\
\hline \multirow{2}{*}{ Sex } & M & \multirow{2}{*}{$\mathrm{n}(\%) *$} & $6(28.6 \%)$ & $5(27.8 \%)$ & $15(68.2 \%)$ & \multirow{2}{*}{0.011} \\
\hline & $\mathrm{F}$ & & $15(71.4 \%)$ & $13(72.2 \%)$ & $7(31.8 \%)$ & \\
\hline \multicolumn{2}{|c|}{ Age } & Mean $+/-\mathrm{SD}^{* *}$ & $66.3+/-7.3$ & $61.1+/-11.2$ & $67.4+/-9.1$ & 0.087 \\
\hline \multicolumn{2}{|c|}{$\begin{array}{l}\text { Follow-up since diagnosis } \\
\text { (months) }\end{array}$} & Mean +/-SD ** & $25.0+/-17.7$ & $29.6+/-16.3$ & $23.5+/-17.1$ & 0.524 \\
\hline \multirow{2}{*}{ Disease stage } & 1 and 2 & \multirow{2}{*}{$\mathrm{n}(\%) *$} & $2(9.5 \%)$ & $12(66.7 \%)$ & $7(31.8 \%)$ & \multirow{2}{*}{0.001} \\
\hline & 3 & & $19(90.5 \%)^{a}$ & $6(33.3 \%)$ & $15(68.2 \%)$ & \\
\hline \multirow{2}{*}{$\begin{array}{l}\text { Adequate fat } \\
\text { intake }\end{array}$} & Increased & \multirow{2}{*}{$\mathrm{n}(\%) *$} & $1(4.8 \%)$ & $4(22.2 \%)$ & $19(86.4 \%)$ & \multirow{2}{*}{$<0.001$} \\
\hline & Yes & & $20(95.2 \%)$ & $14(77.8 \%)$ & $3(13.6 \%)^{a}$ & \\
\hline \multirow{3}{*}{$\begin{array}{c}\text { Adequate } \\
\text { carbohydrate } \\
\text { intake }\end{array}$} & Decreased & \multirow{3}{*}{$\mathrm{n}(\%) *$} & $0(0.0 \%)$ & $1(5.6 \%)$ & $21(95.5 \%)$ & \multirow{3}{*}{$<0.001$} \\
\hline & Yes & & $11(52.4 \%)$ & $17(94.4 \%)$ & $1(4.5 \%)$ & \\
\hline & Increased & & $10(47.6 \%)$ & $0(0.0 \%)$ & $0(0.0 \%)$ & \\
\hline \multirow{2}{*}{$\begin{array}{c}\text { Adequate } \\
\text { protein intake }\end{array}$} & Decreased & \multirow{2}{*}{$\mathrm{n}(\%) *$} & $10(47.6 \%)$ & $6(33.3 \%)$ & $4(18.2 \%)$ & \multirow{2}{*}{0.125} \\
\hline & Yes & & $11(52.4 \%)$ & $12(66.7 \%)$ & $18(81.8 \%)$ & \\
\hline \multicolumn{2}{|c|}{ Saturated fat $(\mathrm{g}) / 1000 \mathrm{kcal}$} & Mean $+/-\mathrm{SD}^{*}$ & $1.0+/-0.2^{\mathrm{a}}$ & $1.2+/-0.2$ & $1.5+/-0.2^{\mathrm{a}}$ & $<0.001$ \\
\hline \multicolumn{2}{|c|}{ Total fiber (g)/1000 kcal } & Mean $+/-\mathrm{SD}^{*}$ & $13.0+/-5.4^{\mathrm{a}}$ & $9.8+/-2.5$ & $6.8+/-1.6^{a}$ & $<0.001$ \\
\hline \multicolumn{2}{|c|}{ Alcohol (g)/1000 kcal } & Mean +/-SD* & $0.7+/-1.2$ & $0.8+/-0.8$ & $2.3+/-2.8^{a}$ & 0.013 \\
\hline \multicolumn{2}{|c|}{ Hemoglobin (g/dl) } & Mean +/-SD ** & $8.1+/-1.7^{a}$ & $9.8+/-2.2$ & $8.0+/-2.0^{a}$ & 0.010 \\
\hline \multicolumn{2}{|c|}{ Alkaline phosphatase (IU/L) } & Mean $+/-\mathrm{SD}^{* *}$ & $114.4+/-31.9$ & $88.1+/-35.8$ & $98.1+/-42.2$ & 0.088 \\
\hline
\end{tabular}


Table 3. Cont.

\begin{tabular}{|c|c|c|c|c|c|c|}
\hline \multirow{2}{*}{\multicolumn{2}{|c|}{ Clinical and Intake Variables }} & \multirow[b]{2}{*}{ Measure } & \multicolumn{3}{|c|}{ Tertiles Of LCD Score } & \multirow[t]{2}{*}{$p$-Values } \\
\hline & & & $\begin{array}{l}\text { First (High Carb } \\
\text { Diet) } n 1=21\end{array}$ & $\begin{array}{c}\text { Second } \\
\text { (Medium Carb } \\
\text { Diet) } n 2=18\end{array}$ & $\begin{array}{l}\text { Third (Low Carb } \\
\text { Diet) } n 3=22\end{array}$ & \\
\hline \multicolumn{2}{|c|}{$\begin{array}{l}\text { Erythrocyte sedimentation rate } \\
\qquad(\mathrm{mm} / \mathrm{hr})\end{array}$} & Mean +/-SD ** & $104.7+/-46.3$ & $102.8+/-40.9$ & $108.1+/-37.5$ & 0.919 \\
\hline \multicolumn{2}{|c|}{ Serum calcium (mg/dL) } & Mean $+/-\mathrm{SD}^{* *}$ & $12.1+/-3.3$ & $11.8+/-2.7$ & $12.2+/-3.1$ & 0.928 \\
\hline \multicolumn{2}{|c|}{ Uric acid (mg/dL) } & Mean $+/-$ SD ** & $7.7+/-1.8^{a}$ & $5.9+/-2.2$ & $6.3+/-1.7$ & 0.006 \\
\hline \multicolumn{2}{|c|}{ Creatinine $(\mathrm{mg} / \mathrm{dL})$} & Mean +/-SD ** & $2.2+/-0.9$ & $1.6+/-1.2$ & $2.4+/-1.5$ & 0.131 \\
\hline \multicolumn{2}{|c|}{ Blood urea nitrogen (mg/dL) } & Mean $+/-\mathrm{SD}^{* *}$ & $58.7+/-21.2$ & $46.9+/-21.0$ & $68.4+/-40.4$ & 0.083 \\
\hline \multicolumn{2}{|c|}{ Albumin (g/L) } & Mean +/ - SD ** & $51.3+/-4.1^{\mathrm{a}}$ & $56.8+/-7.6$ & $54.1+/-5.6$ & 0.018 \\
\hline \multicolumn{2}{|c|}{ Total proteins $(\mathrm{g} / \mathrm{dL})$} & Mean +/ - SD ** & $9.9+/-3.6^{a}$ & $7.2+/-2.4$ & $7.6+/-3.2$ & 0.018 \\
\hline \multicolumn{2}{|c|}{ Beta-2 microglobulin (mg/dl) } & Mean $+/-\mathrm{SD}^{* *}$ & $10.7+/-4.0^{\mathrm{a}}$ & $5.9+/-3.4$ & $8.8+/-4.5$ & 0.002 \\
\hline \multicolumn{2}{|c|}{ C-reactive protein (mg/L) } & Mean $+/-\mathrm{SD}^{* *}$ & $20.3+/-17.7$ & $12.0+/-9.7$ & $18.3+/-13.5$ & 0.179 \\
\hline \multicolumn{2}{|c|}{ Plasmacytes in bone marrow (\%) } & Mean +/-SD ${ }^{* *}$ & $67.5+/-18.5^{a}$ & $44.4+/-23.5$ & $58.2+/-22.7$ & 0.006 \\
\hline \multicolumn{2}{|c|}{$\mathrm{LDH}(\mathrm{U} / \mathrm{L})$} & Mean +/ - SD ** & $300.7+/-104.2$ & $276.8+/-107.3$ & $355.9+/-142.0$ & 0.106 \\
\hline \multicolumn{2}{|c|}{ Fibrinogen (mg/dL) } & Mean $+/-\mathrm{SD}^{* *}$ & $503.1+/-139.9$ & $453.5+/-149.4$ & $473.5+/-157.4$ & 0.580 \\
\hline \multicolumn{2}{|c|}{ D-dimers (ng/mL) } & Mean +/-SD ** & $346.0+/-122.6^{a}$ & $252.4+/-114.3$ & $292.6+/-99.0$ & 0.039 \\
\hline \multirow{4}{*}{$\begin{array}{l}\text { Response } \mathrm{n} \\
(\%)\end{array}$} & Partial remission & & $9(42.9 \%)$ & $8(44.4 \%)$ & $6(27.3 \%)$ & \multirow{4}{*}{0.674} \\
\hline & Total remission & $\mathrm{n}(\%)^{* * *}$ & $4(19.0 \%)$ & $6(33.3 \%)$ & $9(40.9 \%)$ & \\
\hline & Stabile disease & & $5(23.8 \%)$ & $3(16.7 \%)$ & $4(18.2 \%)$ & \\
\hline & $\begin{array}{l}\text { Progressive } \\
\text { disease }\end{array}$ & & $3(14.3 \%)$ & $1(5.6 \%)$ & $3(13.6 \%)$ & \\
\hline \multicolumn{2}{|c|}{ Infections } & $\mathrm{n}(\%) *$ & $6(28.6 \%)$ & $1(5.6 \%)$ & $2(9.1 \%)$ & 0.087 \\
\hline \multicolumn{2}{|c|}{ Myelosuppression } & $\mathrm{n}(\%) *$ & $6(28.6 \%)$ & $1(5.6 \%)$ & $3(13.6 \%)$ & 0.144 \\
\hline \multicolumn{2}{|c|}{ Chronic kidney disease } & $\mathrm{n}(\%) *$ & $8(38.1 \%)$ & $7(38.9 \%)$ & $9(40.9 \%)$ & 0.982 \\
\hline \multicolumn{2}{|c|}{ Peripheral neuropathy } & $\mathrm{n}(\%) *$ & $16(76.2 \%)^{a}$ & $3(16.7 \%)$ & $12(54.5 \%)^{a}$ & 0.001 \\
\hline \multicolumn{2}{|c|}{ Osteoporosis } & $\mathrm{n}(\%) *$ & $7(33.3 \%)$ & $4(22.2 \%)$ & $7(31.8 \%)$ & 0.722 \\
\hline \multicolumn{2}{|c|}{ Depression } & $\mathrm{n}(\%) *$ & $12(57.1 \%)$ & $10(55.6 \%)$ & $11(50.0 \%)$ & 0.888 \\
\hline \multicolumn{2}{|c|}{ Constipation/diarrhea } & $\mathrm{n}(\%) *$ & $7(33.3 \%)$ & $1(5.6 \%)$ & $8(36.4 \%)$ & 0.061 \\
\hline \multicolumn{2}{|c|}{ Therapeutic lines } & Mean +/-SD* & $2.76+/-1.7$ & $3.22+/-1.4$ & $2.86+/-1.5$ & 0.628 \\
\hline
\end{tabular}

Notes: Data are presented as mean +/-SD, or as n (\%), as appropriate. ${ }^{*}$ Kruskal-Wallis test; ${ }^{* *}$ ANOVA; ${ }^{* * *}$ chi-square test. ${ }^{\text {a }}$ Superscript letter denotes a significant difference, as compared to the second tertile using Mann-Whitney test with Bonferroni adjustment, or post hoc tests with Sidak adjustment, as appropriate. $p$-values in bold are statistically significant. Abbreviations: $\mathrm{SD}=$ standard deviation, $\mathrm{n}=$ number of participants, LCD = low carbohydrate diet.

Table 4 shows the percentages of nutrition knowledge score per each section and the total score. Compared to the previously published knowledge scores, calculated for the Romanian population [20], Sections 1,3 and 4 scores were lower, but the score for Section 2 and the total score were similar. The sub-scores per section and the total score did not differ when using demographic and clinical factors (Table 4). 
Table 4. Nutrition knowledge score percentages per sections and total score ( $\mathrm{N}=61$ participants).

\begin{tabular}{|c|c|c|c|c|c|c|}
\hline \multicolumn{2}{|c|}{ Factors } & $\begin{array}{c}\text { Section } 1 \\
\text { Achievement } \\
\text { (\%)Expert Rec- } \\
\text { ommendations }\end{array}$ & $\begin{array}{c}\text { Section } 2 \\
\text { Achievement } \\
(\%) \text { Food } \\
\text { Groups }\end{array}$ & $\begin{array}{c}\text { Section } 3 \\
\text { Achievement } \\
\text { (\%) Healthy } \\
\text { Food Choices }\end{array}$ & $\begin{array}{l}\text { Section } 4 \\
\text { Achievement } \\
\text { Diet, Disease } \\
\text { and Weight } \\
\text { Associations }\end{array}$ & $\begin{array}{c}\text { Total Score } \\
\text { Achievement } \\
\quad(\%)\end{array}$ \\
\hline \multicolumn{2}{|c|}{ Median achievement } & $64.0+/-9.4 *$ & $63.5+/-9.2$ & $71.6+/-13.9 *$ & $65.0+/-11.2 *$ & $65.2+/-7.1$ \\
\hline \multirow{2}{*}{ Sex } & M & $63.7+/-10.3$ & $63.1+/-8.4$ & $71.6+/-16.2$ & $67.2+/-9.3$ & $65.5+/-7.7$ \\
\hline & $\mathrm{F}$ & $64.3+/-8.8$ & $63.7+/-9.8$ & $71.6+/-12.2$ & $63.4+/-12.3$ & $64.9+/-6.8$ \\
\hline \multirow{2}{*}{ Age category } & $\leq 65$ years & $65.9+/-10.4$ & $63.8+/-10.5$ & $73.4+/-13.6$ & $67.3+/-11.0$ & $66.5+/-7.5$ \\
\hline & $>65$ years & $62.0+/-7.9$ & $63.1+/-7.8$ & $69.7+/-14.3$ & $62.7+/-11.1$ & $63.8+/-6.6$ \\
\hline \multirow[t]{2}{*}{ Education } & $\begin{array}{c}\text { High school or } \\
\text { less }\end{array}$ & $61.8+/-10.2$ & $63.9+/-9.0$ & $73.6+/-14.6$ & $65.9+/-10.1$ & $65.4+/-7.4$ \\
\hline & $\begin{array}{c}\text { At least college } \\
\text { degree }\end{array}$ & $66.5+/-7.9$ & $63.0+/-9.5$ & $69.5+/-13.1$ & $64.0+/-12.4$ & $64.9+/-7.0$ \\
\hline \multirow{3}{*}{$\begin{array}{l}\text { Living with } \\
\text { underage } \\
\text { individuals }\end{array}$} & Yes & $64.3+/-9.3$ & $63.7+/-9.4$ & $71.0+/-13.6$ & $65.0+/-11.2$ & $65.2+/-7.4$ \\
\hline & No & $61.1+/-10.5$ & $61.6+/-6.4$ & $76.9+/-16.9$ & $65.1+/-12.3$ & $64.6+/-4.9$ \\
\hline & First (high carb) & $62.4+/-9.1$ & $62.8+/-8.0$ & $73.3+/-12.6$ & $61.9+/-12.1$ & $64.1+/-5.2$ \\
\hline \multirow{2}{*}{$\begin{array}{c}\text { Percentile } \\
\text { group of LCD } \\
\text { score }\end{array}$} & $\begin{array}{l}\text { Second } \\
\text { (medium) }\end{array}$ & $67.0+/-8.8$ & $63.1+/-11.7$ & $68.8+/-13.6$ & $65.3+/-11.4$ & $65.3+/-8.6$ \\
\hline & $\begin{array}{l}\text { Third (Low } \\
\text { carb) }\end{array}$ & $63.1+/-9.9$ & $64.4+/-8.2$ & $72.4+/-15.7$ & $67.7+/-9.7$ & $66.1+/-7.6$ \\
\hline \multirow{2}{*}{ Anemia } & No & $66.7+/-7.0$ & $60.2+/-13.1$ & $70.5+/-13.2$ & $61.9+/-6.0$ & $63.4+/-7.8$ \\
\hline & Yes & $63.7+/-9.6$ & $63.8+/-8.7$ & $71.7+/-14.1$ & $65.4+/-11.6$ & $65.4+/-7.1$ \\
\hline \multirow{2}{*}{ Hypercalcemia } & No & $65.5+/-6.4$ & $62.9+/-9.4$ & $73.2+/-13.6$ & $63.7+/-9.0$ & $65.2+/-6.8$ \\
\hline & Yes & $62.7+/-11.4$ & $64.0+/-9.0$ & $70.2+/-14.3$ & $66.2+/-12.9$ & $65.2+/-7.6$ \\
\hline \multirow{2}{*}{$\begin{array}{l}\text { Chronic kidney } \\
\text { disease }\end{array}$} & No & $65.3+/-8.3$ & $63.7+/-9.4$ & $73.0+/-13.3$ & $66.8+/-10.2$ & $66.2+/-6.8$ \\
\hline & Yes & $62.0+/-10.7$ & $63.1+/-9.0$ & $69.6+/-15.0$ & $62.3+/-12.3$ & $63.6+/-7.5$ \\
\hline \multirow{2}{*}{ Infections } & No & $65.2+/-8.9$ & $64.4+/-9.1$ & $72.0+/-13.6$ & $65.3+/-11.5$ & $65.9+/-7.2$ \\
\hline & Yes & $57.4+/-9.6$ & $58.3+/-8.4$ & $69.2+/-16.3$ & $63.5+/-9.5$ & $61.0+/-5.0$ \\
\hline
\end{tabular}

Notes: Data is presented as mean $+/-$ SD. * Statistically significant lower achievement score, when compared to the population median, with the following values: Section $1-66.7 \%$, Section $2-63.8 \%$, Section 3-76.9\%, Section 4-71.4\%, total score-65.9\% [20]; between factors comparisons with independent samples $t$-test or ANOVA, as appropriate.

\section{Discussion}

To the best of our knowledge, neither the LCD score nor the nutrition knowledge has been assessed for patients diagnosed with MM. LCD score has been used for several years in relation to the risk of chronic diseases [18,19] and mortality [21]. Nutrition knowledge was previously associated with healthier food choices [22-24] and is a necessary, but not a sufficient, component of behavioral change.

By tertiles of energy, the energy-adjusted intake of nutrients did not reach statistical significance, meaning that the increase in energy is not due to a particular macronutrient source of energy. A higher LCD score, associated with a diet lower in carbohydrates, was observed in the lowest tertile of energy (Table 3). Proportions of the adequacy of protein intake showed a linear trend, with the lowest tertile being associated with the lowest proportion of adequacy of intake and reaching up to $100 \%$ in the third LCD tertile.

Similar to other populational studies [25], by tertiles of LCD score, women are more likely than men to have higher intakes of carbohydrates. Low carbohydrate diets have become popular due to short-term weight loss, with better results when associated with high protein diets [26,27], but recent research is inconsistent regarding the long-term 
effects on diabetes [28,29]. High mortality rates and increased risk of chronic diseases were reported among people consuming low carbohydrate diets, especially when low carbohydrate diets were accompanied by high fat intake, especially saturated fat, and a low fiber intake [18,19,21,30-32]. In our sample (Table 3), low carbohydrate diets were associated with high saturated fat and alcohol intake and low fiber intake, situating our patients in the high-risk group. Yet, some clinical indicators, such as a significantly lower hemoglobin level and higher prevalence of reported peripheral neuropathy, were reported in our low carbohydrate-high saturated fat tertile.

Then again, high carbohydrate diets are also associated with high morbidity and mortality rates [33-35]. As demonstrated by our small sample, compared to the medium tertile of LCD score, which has the highest proportions of macronutrient adequacy $(94.4 \%$ for carbohydrate, $77.8 \%$ for fat and $66.7 \%$ for protein), the high carbohydrate tertile, with $52.4 \%$ for carbohydrate, $95.2 \%$ for fat and $52.4 \%$ for protein, has the worst clinical indicators (Table 3). Anemia has a broad implication, i.e., the low hemoglobin and hematocrit, and induces or aggravates hypoxia, impacts the cardiovascular system, is associated with poor quality of life and performance and impairs daily activity. Considering that most MM patients are elderly, the clinical aspects previously presented may be even more important [36]. Anemia is often associated with and aggravated by chronic kidney disease [37].

Several hemostatic and thrombotic anomalies have been reported in patients with MM, with D-dimers being the most commonly reported prothrombotic marker [38]. D-dimers are associated with poor prognosis in patients with cancer [39]. Recent research has shown that hypercalcemia and bone disease are significantly associated with a worse prognosis [40]. The International Staging System, which is based on serum beta-2 microglobulin and albumin levels, is the most widely adopted in multiple myeloma and is also correlated with the prognosis of the disease [40]. Assessment of bone marrow involvement by malignant plasma cells is an important element in the diagnosis and follow-up of patients with multiple myeloma and other plasma cell dyscrasias [40,41].

Patients with MM suffer diagnostic delays due to the complex nature of the disease and non-specificity of symptoms [42-44]. Often, due to these delays, patients are diagnosed in the late stage of disease, associated with poor prognosis [40]. In our experience, patients are often diagnosed in stage 3 disease, and this is reflected in our sample by shorter time to follow-up, compared to patients in stages 1 and 2 (Table 1). Patients in disease stage 3 are more likely to report either high carbohydrate intake or low carbohydrate intake, the latter not reaching statistical significance after Bonferroni correction (Table 3). In our sample, patients from the high carbohydrate diet tertile have lower hemoglobin and albumin levels and higher D-dimers, calcium, uric acid, percentage of plasmacytes in the bone marrow and beta- 2 microglobulin levels, when compared to patients from the medium carbohydrate tertile. Since our study is cross-sectional, we cannot assume causality, only association. Further research will be able to clarify some of these mechanisms observed between clinical outcomes and patterns of nutrient intake, and the relationships between clinical indicators of disease status.

There are several proposed mechanisms to explain the association of anemia with $\mathrm{MM}$, and the most important seems to be inadequate erythropoietin (EPO) production related to inflammatory cytokines [45] and the high levels of hepcidin, the iron regulatory hormone that works by restricting the iron supply for erythropoiesis [46]. Several dietary factors can aggravate anemia, and phytates, fiber and starches were considered to have inhibitory effects on iron absorption [47]. In a 10-year retrospective study in patients with MM [48], low folate levels were associated with lower levels of hemoglobin. High BMI has a protective effect for anemia, but even in this population, increasing the dietary fat/carbohydrate ratio increases the risk of anemia [49].

Purine-rich foods, high protein intake, alcohol and fructose are known risk factors for increasing the levels of uric acid [50]. Fructose directly regulates uric acid production by increasing ATP degradation to AMP, a uric acid precursor [50]. Intervention studies 
have shown that the relationship between carbohydrate intake and uric acid production is mediated by the glycemic index of food, with the intake of food with low glycemic index lowering the levels of uric acid [51].

Total nutrition knowledge scores (Table 4) were low, but were similar to those of recent research [20] that used the same instrument. In the sections Expert recommendations, Healthy food choices and Diet, disease and weight associations, our sample scored lower than the general population, so this could represent a starting point in targeted diet interventions. The nutrition knowledge score did not differ by demographics, intake or clinical indicators, though some trends were observed, but did not reach statistical significance.

Patients with MM may gain benefits if they receive nutritional counseling and healthy eating guidance, in order to learn how to sustain a balanced diet, because nutritional needs change during the course of the disease and survivorship [52]. A study by Lee et al. [8] suggests that increased scores in different patterns of intake, such as in the Alternate Healthy Eating Index (AHEI)-2010, Alternate Mediterranean Diet or Dietary Approaches to Stop Hypertension were associated with better prognosis and survival in patients with MM. Intermittent fasting has been proposed as an alternative option in cancer therapy due to autophagy, but has been controversial [53-55].

Epigenetic mechanisms via DNA methylation, histone modifications and non-coding RNAs were found to be associated with better evolution and therapeutic response in patients with MM [56]. Although adequate intake of methyl donors such as folate, methionine, choline and vitamins B2, B6, B12 are likely to impact epigenetic mechanisms [57], better understanding of the different polymorphisms from the genes involved in one-carbon metabolism could shed new light on nutrition counseling for patients with MM [58,59].

A major limitation of our study is the small number of participants since recruitment occurred during the second wave of the COVID-19 pandemic. Secondly, participants were all recruited in a single university clinic, thus the results cannot be generalized. A larger multicentric sample would be necessary to be able to generalize these findings. The participants were volunteers, which might have biased selection toward individuals more interested in food, diet and health and with a genuine interest in these matters, as compared to those who declined participation. Since the current study did not receive external funding, not all participants received metabolic panel analyses. Subsequent studies should plan to overcome this limitation.

\section{Conclusions}

Stage 3 disease in patients with MM is associated with either a high carbohydrate or low carbohydrate-high saturated fat diet. Other clinical indicators, besides beta- 2 microglobulin, which have been related to poor prognosis, are associated in our sample with a high carbohydrate diet. During disease treatment and survivorship, the nutritional needs of MM patients change, thus a better knowledge of foods and nutritional value could encourage them to make healthier decisions that might extend survival.

Author Contributions: Conceptualization, E.B. and H.I.; methodology, C.L.S., E.B., H.I., I.I.; software, C.L.S. and S.P.; validation, E.B., S.P., C.P., O.P., M.S. and R.T.; formal analysis, C.L.S.; investigation, E.B., C.P., O.P., M.S. and R.T.; resources, I.I. and H.I.; data curation, E.B. and C.L.S.; writing-original draft preparation, E.B.; writing-review and editing, C.L.S. and H.I.; supervision, H.I. All authors have read and agreed to the published version of the manuscript.

Funding: This research received no external funding.

Institutional Review Board Statement: The study was approved by the Ethics Committee of the "Victor Babes" University of Medicine and Pharmacy (6/2019), Timisoara, Romania, and conducted in accordance with the Declaration of Helsinki.

Informed Consent Statement: Written informed consent was obtained from all subjects involved in the study.

Data Availability Statement: Raw data for variables of the cohort are available on request. 
Conflicts of Interest: The authors declare no conflict of interest.

\section{References}

1. Cancer Today. Available online: http://gco.iarc.fr/today/home (accessed on 9 February 2021).

2. Padala, S.; Barsouk, A.; Barsouk, A.; Rawla, P.; Vakiti, A.; Kolhe, R.; Kota, V.; Ajebo, G. Epidemiology, Staging, and Management of Multiple Myeloma. Med. Sci. 2021, 9, 3. [CrossRef]

3. Usmani, S.Z.; Hoering, A.; Cavo, M.; Miguel, J.S.; Goldschimdt, H.; Hajek, R.; Turesson, I.; Lahuerta, J.J.; Attal, M.; Barlogie, B.; et al. Clinical predictors of long-term survival in newly diagnosed transplant eligible multiple myeloma-An IMWG Research Project. Blood Cancer J. 2018, 8, 1-7. [CrossRef]

4. Thordardottir, M.; Lindqvist, E.K.; Lund, S.H.; Costello, R.; Burton, D.; Steingrimsdottir, L.; Korde, N.; Mailankody, S.; Eiriksdottir G.; Launer, L.J.; et al. Dietary intake is associated with risk of multiple myeloma and its precursor disease. PLoS ONE 2018, 13, e0206047. [CrossRef] [PubMed]

5. Fritschi, L.; Ambrosini, G.L.; Kliewer, E.V.; Johnson, K.C. Canadian Cancer Registries Epidemiologic Research Group. Dietary fish intake and risk of leukaemia, multiple myeloma, and non-Hodgkin lymphoma. Cancer Epidemiol. Prev. Biomark. 2004, 13, 532-537.

6. Andreotti, G.; Birmann, B.; De Roos, A.J.; Spinelli, J.; Cozen, W.; Camp, N.J.; Moysich, K.; Chiu, B.; Steplowski, E.; Krzystan, J.; et al. A Pooled Analysis of Alcohol Consumption and Risk of Multiple Myeloma in the International Multiple Myeloma Consortium. Cancer Epidemiol. Biomark. Prev. 2013, 22, 1620-1627. [CrossRef] [PubMed]

7. Schernhammer, E.S.; Bertrand, K.; Birmann, B.M.; Sampson, L.; Willett, W.C.; Feskanich, D. Consumption of artificial sweetenerand sugar-containing soda and risk of lymphoma and leukemia in men and women. Am. J. Clin. Nutr. 2012, 96, 1419-1428 [CrossRef] [PubMed]

8. Lee, D.H.; Fung, T.T.; Tabung, F.K.; Marinac, C.R.; Devore, E.E.; Rosner, B.A.; Ghobrial, I.M.; Colditz, G.A.; Giovannucci, E.L.; Birmann, B.M. Prediagnosis dietary pattern and survival in patients with multiple myeloma. Int. J. Cancer 2020, 147, 1823-1830 [CrossRef]

9. Viechtbauer, W.; Smits, L.; Kotz, D.; Budé, L.; Spigt, M.; Serroyen, J.; Crutzen, R. A simple formula for the calculation of sample size in pilot studies. J. Clin. Epidemiol. 2015, 68, 1375-1379. [CrossRef]

10. Dimopoulos, M.A.; Moreau, P.; Terpos, E.; Mateos, M.V.; Zweegman, S.; Cook, G. Multiple myeloma: EHA-ESMO Clinical Practice Guidelines for diagnosis, treatment and follow-up. Ann. Oncol. 2021, 32, 309-322. [CrossRef]

11. Baumgarten, M.; Gehr, T. Chronic kidney disease: Detection and evaluation. Am. Fam. Physician 2011, 84, 1138-1148.

12. Jeremiah, M.P.; Unwin, B.K.; Greenawald, M.H.; Casiano, V.E. Diagnosis and Management of Osteoporosis. Am. Fam. Physician. 2015, 92, 261-268. [PubMed]

13. Ng, C.W.M.; How, C.H.; Ng, Y.P. Major depression in primary care: Making the diagnosis. Singap. Med. J. 2016, 57, 591-597. [CrossRef]

14. Haftenberger, M.; Heuer, T.; Heidemann, C.; Kube, F.; Krems, C.; Mensink, G.B. Relative validation of a food frequency questionnaire for national health and nutrition monitoring. Nutr. J. 2010, 9, 36. [CrossRef] [PubMed]

15. Serban, C.; Banu, A.; Putnoky, S.; Butica, S.I.; Niculescu, M.D.; Putnoky, S. Relative validation of a four weeks retrospective food frequency questionnaire versus 7-day paper-based food records in estimating intake of energy and nutrients in adults. Nutr. Diet. Suppl. Dove Press 2021, in press.

16. U.S. Food and Drug Administration (FDA). Guidance for Industry: Guidelines for Determining Metric Equivalents of Household Measures. 2020. Available online: https:/ / www.fda.gov/regulatory-information/search-fda-guidance-documents/guidanceindustry-guidelines-determining-metric-equivalents-household-measures (accessed on 19 February 2021).

17. Dietary Reference Values for nutrients Summary report. EFSA Support Publ. 2017, 14, e15121E.

18. Halton, T.L.; Willett, W.C.; Liu, S.; Manson, J.E.; Albert, C.; Rexrode, K.; Hu, F.B. Low-Carbohydrate-Diet Score and the Risk of Coronary Heart Disease in Women. N. Engl. J. Med. 2006, 355, 1991-2002. [CrossRef] [PubMed]

19. De Koning, L.; Fung, T.T.; Liao, X.; Chiuve, S.E.; Rimm, E.B.; Willett, W.C. Low-carbohydrate diet scores and risk of type 2 diabetes in men. Am. J. Clin. Nutr. 2011, 93, 844-850. [CrossRef]

20. Putnoky, S.; Banu, A.M.; Moleriu, L.C.; Putnoky, S.; Șerban, D.M.; Niculescu, M.D.; Șerban, C.L. Reliability and validity of a General Nutrition Knowledge Questionnaire for adults in a Romanian population. Eur. J. Clin. Nutr. 2020, 74, 1576-1584. [CrossRef]

21. Fung, T.T.; van Dam, R.M.; Hankinson, S.E.; Stampfer, M.; Willett, W.C.; Hu, F.B. Low-carbohydrate diets and all-cause and cause-specific mortality: Two cohort studies. Ann. Intern. Med. 2010, 153, 289-298. [CrossRef]

22. Wardle, J.; Parmenter, K.; Waller, J. Nutrition knowledge and food intake. Appetite 2000, 34, 269-275. [CrossRef]

23. Hill, C.R.; Blekkenhorst, L.C.; Radavelli-Bagatini, S.; Sim, M.; Woodman, R.J.; Devine, A.; Shaw, J.E.; Hodgson, J.M.; Daly, R.M.; Lewis, J.R. Fruit and Vegetable Knowledge and Intake within an Australian Population: The AusDiab Study. Nutrients 2020, 12, 3628. [CrossRef] [PubMed]

24. McKinnon, L.; Giskes, K.; Turrell, G. The contribution of three components of nutrition knowledge to socio-economic differences in food purchasing choices. Public Health Nutr. 2013, 17, 1814-1824. [CrossRef] [PubMed]

25. Leblanc, V.; Bégin, C.; Corneau, L.; Dodin, S.; Lemieux, S. Gender differences in dietary intakes: What is the contribution of motivational variables? J. Hum. Nutr. Diet. 2014, 28, 37-46. [CrossRef] [PubMed] 
26. Clifton, P.; Condo, D.; Keogh, J. Long term weight maintenance after advice to consume low carbohydrate, higher protein diets-A systematic review and meta analysis. Nutr. Metab. Cardiovasc. Dis. 2014, 24, 224-235. [CrossRef]

27. Maula, A.; Kai, J.; Woolley, A.K.; Weng, S.; Dhalwani, N.; Griffiths, F.E.; Khunti, K.; Kendrick, D. Educational weight loss interventions in obese and overweight adults with type 2 diabetes: A systematic review and meta-analysis of randomized controlled trials. Diabet. Med. 2019, 37, 623-635. [CrossRef] [PubMed]

28. Larsen, R.N.; Mann, N.J.; MacLean, E.; Shaw, J.E. The effect of high-protein, low-carbohydrate diets in the treatment of type 2 diabetes: A 12 month randomised controlled trial. Diabetologia 2011, 54, 731-740. [CrossRef] [PubMed]

29. Huntriss, R.; Campbell, M.; Bedwell, C. The interpretation and effect of a low-carbohydrate diet in the management of type 2 diabetes: A systematic review and meta-analysis of randomised controlled trials. Eur. J. Clin. Nutr. 2017, 72, 311-325. [CrossRef]

30. Noto, H.; Goto, A.; Tsujimoto, T.; Noda, M. Low-Carbohydrate Diets and All-Cause Mortality: A Systematic Review and Meta-Analysis of Observational Studies. PLoS ONE 2013, 8, e55030. [CrossRef]

31. Lagiou, P.; Sandin, S.; Lof, M.; Trichopoulos, D.; Adami, H.-O.; Weiderpass, E. Low carbohydrate-high protein diet and incidence of cardiovascular diseases in Swedish women: Prospective cohort study. BMJ 2012, 344, e4026. [CrossRef] [PubMed]

32. Fechner, E.; Smeets, E.T.; Schrauwen, P.; Mensink, R.P. The Effects of Different Degrees of Carbohydrate Restriction and Carbohydrate Replacement on Cardiometabolic Risk Markers in Humans-A Systematic Review and Meta-Analysis. Nutrients 2020, 12, 991. [CrossRef]

33. Dehghan, M.; Mente, A.; Zhang, X.; Swaminathan, S.; Li, W.; Mohan, V.; Iqbal, R.; Kumar, R.; Wentzel-Viljoen, E.; Rosengren, A.; et al. Associations of fats and carbohydrate intake with cardiovascular disease and mortality in 18 countries from five continents (PURE): A prospective cohort study. Lancet 2017, 390, 2050-2062. [CrossRef]

34. Seidelmann, S.B.; Claggett, B.; Cheng, S.; Henglin, M.; Shah, A.; Steffen, L.M.; Folsom, A.R.; Rimm, E.B.; Willett, W.C.; Solomon, S.D. Dietary carbohydrate intake and mortality: A prospective cohort study and meta-analysis. Lancet Public Health 2018, 3 , e419-e428. [CrossRef]

35. Mente, A.; Yusuf, S. Evolving evidence about diet and health. Lancet Public Health 2018, 3, e408-e409. [CrossRef]

36. Wouters, H.J.; Van Der Klauw, M.M.; De Witte, T.; Stauder, R.; Swinkels, D.W.; Wolffenbuttel, B.H.; Huls, G. Association of anemia with health-related quality of life and survival: A large population-based cohort study. Haematologica 2018, 104, 468-476. [CrossRef] [PubMed]

37. Banaszkiewicz, M.; Małyszko, J.; Vesole, D.H.; Woziwodzka, K.; Jurczyszyn, A.; Żórawski, M.; Krzanowski, M.; Małyszko, J.; Batko, K.; Kuźniewski, M.; et al. New Biomarkers of Ferric Management in Multiple Myeloma and Kidney Disease-Associated Anemia. J. Clin. Med. 2019, 8, 1828. [CrossRef] [PubMed]

38. Gogia, A.; Sikka, M.; Sharma, S.; Rusia, U. Hemostatic Abnormalities in Multiple Myeloma Patients. Asian Pac. J. Cancer Prev. 2012, 19, 127-130.

39. Ay, C.; Dunkler, D.; Pirker, R.; Thaler, J.; Quehenberger, P.; Wagner, O.; Zielinski, C.; Pabinger, I. High D-dimer levels are associated with poor prognosis in cancer patients. Haematologica 2012, 97, 1158-1164. [CrossRef] [PubMed]

40. Nakaya, A.; Fujita, S.; Satake, A.; Nakanishi, T.; Azuma, Y.; Tsubokura, Y.; Hotta, M.; Yoshimura, H.; Ishii, K.; Ito, T.; et al. Impact of CRAB Symptoms in Survival of Patients with Symptomatic Myeloma in Novel Agent Era. Hematol. Rep. 2017, 9, 6887. [CrossRef]

41. Cazaubiel, T.; Mulas, O.; Montes, L.; Schavgoulidze, A.; Avet-Loiseau, H.; Corre, J.; Perrot, A. Risk and Response-Adapted Treatment in Multiple Myeloma. Cancers 2020, 12, 3497. [CrossRef]

42. Lyratzopoulos, G.; Neal, R.D.; Barbiere, J.M.; Rubin, G.P.; Abel, G. Variation in number of general practitioner consultations before hospital referral for cancer: Findings from the 2010 National Cancer Patient Experience Survey in England. Lancet Oncol. 2012, 13, 353-365. [CrossRef]

43. Lyratzopoulos, G.; Wardle, J.; Rubin, G. Rethinking diagnostic delay in cancer: How difficult is the diagnosis? BMJ 2014, 349, g7400. [CrossRef] [PubMed]

44. Koshiaris, C. Methods for reducing delays in the diagnosis of multiple myeloma. Int. J. Hematol. Oncol. 2019, 8, IJH13. [CrossRef] [PubMed]

45. Mittelman, M. The Implications of Anemia in Multiple Myeloma. Clin. Lymphoma 2003, 4, S23-S29. [CrossRef] [PubMed]

46. Maes, K.; Nemeth, E.; Roodman, G.D.; Huston, A.; Esteve, F.; Freytes, C.; Callander, N.; Katodritou, E.; Tussing-Humphreys, L.; Rivera, S.; et al. In anemia of multiple myeloma, hepcidin is induced by increased bone morphogenetic protein 2. Blood 2010, 116, 3635-3644. [CrossRef] [PubMed]

47. De Rozo, M.P.; Van Campen, D.; Miller, D.D. Effects of some carbohydrates on iron absorption. Arch. Latinoam. Nutr. 1986, 36, 688-700. [PubMed]

48. Wongrakpanich, S.; George, G.; Chaiwatcharayut, W.; Candelario, N.; Mittal, V.; Pomerantz, S.; Varadi, G. Frequency of folate deficiency in multiple myeloma patients: A 10-year retrospective study. Int. J. Lab. Hematol. 2015, 38, e19-e22. [CrossRef]

49. Chang, J.-S.; Chen, Y.-C.; Owaga, E.; Palupi, K.C.; Pan, W.-H.; Bai, C.-H. Interactive Effects of Dietary Fat/Carbohydrate Ratio and Body Mass Index on Iron Deficiency Anemia among Taiwanese Women. Nutrients 2014, 6, 3929-3941. [CrossRef]

50. Rho, Y.H.; Zhu, Y.; Choi, H.K. The Epidemiology of Uric Acid and Fructose. Semin. Nephrol. 2011, 31, 410-419. [CrossRef]

51. Juraschek, S.P.; McAdams-DeMarco, M.; Gelber, A.C.; Sacks, F.M.; Appel, L.J.; White, K.J.; Miller, E.R. Effects of Lowering Glycemic Index of Dietary Carbohydrate on Plasma Uric Acid Levels: The OmniCarb Randomized Clinical Trial. Arthritis Rheumatol. 2015, 68, 1281-1289. [CrossRef] 
52. Rock, C.L.; Doyle, C.; Demark-Wahnefried, W.; Meyerhardt, J.; Courneya, K.S.; Schwartz, A.L. Nutrition and physical activity guidelines for cancer survivors. CA Cancer J. Clin. 2012, 62, 242-274. [CrossRef]

53. Chen, N.; Karantza, V. Autophagy as a therapeutic target in cancer. Cancer Biol. Ther. 2011, 11, 157-168. [CrossRef] [PubMed]

54. Galluzzi, L.; Pedro, J.M.B.-S.; Levine, B.; Green, D.R.; Kroemer, G. Pharmacological modulation of autophagy: Therapeutic potential and persisting obstacles. Nat. Rev. Drug Discov. 2017, 16, 487-511. [CrossRef] [PubMed]

55. Antunes, F.; Erustes, A.G.; Costa, A.J.; Nascimento, A.C.; Bincoletto, C.; Ureshino, R.P.; Pereira, G.J.S.; Smaili, S.S. Autophagy and intermittent fasting: The connection for cancer therapy? Clinics 2018, 73. [CrossRef] [PubMed]

56. Alzrigat, M.; Párraga, A.A.; Jernberg-Wiklund, H. Epigenetics in multiple myeloma: From mechanisms to therapy. Semin. Cancer Biol. 2018, 51, 101-115. [CrossRef] [PubMed]

57. Kadayifci, F.Z.; Zheng, S.; Pan, Y.-X. Molecular Mechanisms Underlying the Link between Diet and DNA Methylation. Int. J. Mol. Sci. 2018, 19, 4055. [CrossRef]

58. Zintzaras, E.; Giannouli, S.; Rodopoulou, P.; Voulgarelis, M. The role of MTHFR gene in multiple myeloma. J. Hum. Genet. 2008, 53, 499-507. [CrossRef]

59. Weiner, A.S.; Boyarskikh, U.A.; Voronina, E.N.; Mishukova, O.V.; Filipenko, M.L. Methylenetetrahydrofolate reductase C677T and methionine synthase A2756G polymorphisms influence on leukocyte genomic DNA methylation level. Gene 2014, 533, 168-172. [CrossRef] 